\title{
RISK-INFORMED DECISION-MAKING IN THE PRESENCE OF EPISTEMIC UNCERTAINTY
}

\section{Didier Dubois ${ }^{1}$, Dominique Guyonnet ${ }^{2 *}$}

\section{Accepted for publication in "International Journal of General Systems"}

\author{
1 IRIT, CNRS and University of Toulouse, France \\ 2 BRGM, Orléans, France \\ * Address correspondence to Dr. Dominique Guyonnet, BRGM, 3 Av. C. Guillemin, BP \\ 36009, 45060 Orléans cedex 2, France; tel +33 2386438 17; d.guyonnet@brgm.fr
}

\begin{abstract}
An important issue in risk analysis is the distinction between epistemic and aleatory uncertainties. In this paper, the use of distinct representation formats for aleatory and epistemic uncertainties is advocated, the latter being modelled by sets of possible values. Modern uncertainty theories based on convex sets of probabilities are known to be instrumental for hybrid representations where aleatory and epistemic components of uncertainty remain distinct. Simple uncertainty representation techniques based on fuzzy intervals and p-boxes are used in practice. This paper outlines a risk analysis methodology from elicitation of knowledge about parameters to decision. It proposes an elicitation methodology where the chosen representation format depends on the nature and the amount of available information. Uncertainty propagation methods then blend Monte-Carlo simulation and interval analysis techniques. Nevertheless, results provided by these techniques, often in terms of probability intervals, may be too complex to interpret for a decision-maker and we therefore propose to compute a unique indicator of the likelihood of risk, called confidence index. It explicitly accounts for the decision-maker's attitude in the face of ambiguity. This step takes place at the end of the risk analysis process, when no further collection of evidence is possible that might reduce the ambiguity due to epistemic uncertainty. This last feature stands in contrast with the Bayesian methodology, where epistemic uncertainties on input parameters are modelled by single subjective probabilities at the beginning of the risk analysis process.
\end{abstract}

KEY WORDS: Epistemic uncertainty, risk analysis, uncertainty propagation, decision-making 


\section{INTRODUCTION}

With the quest for sustainable development, the notion of risk is increasingly present in our collective psyche, as can be seen in public regulations regarding the management of water (e.g. OJEC 2000), soil (CEC 2006) or waste (OJEC 2008). Risk in such contexts can be defined as the combination of the likelihood of occurrence of an undesirable event and the severity of the damage that can be caused by the event (e.g., BSI 2007). In recent years, a clearer understanding of what can be expected from environmental risk assessments has emerged, with a shift from "risk-based" management (e.g., Vegter 2001) to "risk-informed" management (Burton et al. 2008; Pollard et al. 2002), whereby risk assessment is but one component of the decision-making process, to be combined with other criteria from a variety of fields, e.g., environmental, economic and social. Such a shift is primarily the result of a better awareness that decision-making in the environmental field is a multi-factor process and of the limitations of risk assessment due in particular to inherent uncertainties.

In the last 10 years or so, the treatment of uncertainty in risk assessments has witnessed a shift of paradigm with the increasing awareness of the fundamental difference between stochastic and epistemic uncertainties (Hoffman and Hammonds 1994, Ferson 1996, Ferson and Ginzburg 1996, Guyonnet et al. 1999, Helton et al. 2004, Colyvan 2008). Stochastic (or aleatory) uncertainty arises from random variability related to natural processes such as the heterogeneity of population or the fluctuations of a quantity with time. Epistemic uncertainty arises from the incomplete/imprecise nature of available information. The pervasive confusion between these two types of uncertainties has been one of the most serious shortcomings in risk assessment.

While stochastic uncertainty is adequately addressed using classical probability theory, several uncertainty theories have been developed in order to explicitly handle incomplete/imprecise information (see for instance the survey by Dubois and Prade 2009). Such developments in uncertainty theories provide new tools for faithfully representing the kind of poor information collected by practitioners in the environmental field. As a result, some risk analysts have felt the need to develop original computation schemes for jointly propagating information tainted with epistemic and stochastic uncertainties. Such joint propagation methods have been applied by a number of authors. For instance, Li et al. (2007) used an integrated fuzzy-stochastic approach in the assessment of the risk of groundwater contamination by hydrocarbons. Baraldi and Zio (2008) used a combined Monte Carlo and possibilistic (fuzzy) approach to propagate uncertainties in an event tree analysis of accident 
sequences in a nuclear power plant in Taïwan. Li et al. (2008) used a fuzzy-stochastic modelling approach for estimating health risks from air pollution. Baccou et al. (2008) applied joint propagation methods for assessing the risk of radionuclide migration in the environment. Kentel and Aral (2005) compared 2D Monte Carlo and joint fuzzy and Monte Carlo propagation for calculating health risks, while Kentel (2006) applied such joint methods to groundwater resource management. Bellenfant et al. (2008) used another method (referred to as IRS in section 4 of this paper) to quantify risks of $\mathrm{CO}_{2}$ leakage following injection into deep geological deposits.

Results of joint propagation methods, such as those developed by these authors, can typically be expressed by means of special “families” of probability distributions, as opposed to single distributions. They are delimited by an upper bound (a plausibility function in evidence theory) and a lower bound (a belief function) of the probability that risk might exceed or not a certain threshold. Compared to the result of a classical Monte Carlo analysis performed using subjective probability distributions for modelling incomplete/imprecise information, hybrid methods do not yield a unique estimate of the probability that risk should exceed or not a certain threshold. Although the very aim of these joint propagation methods is to promote consistency with available information and avoid assumptions of Bayesian methods (Dubois et al. 1996, Ben-Haim 2006), the use of probability intervals may become an impediment at the decision-making stage, since decision-makers may not feel comfortable with the notion of an imprecise probability of exceeding a threshold. In the Bayesian tradition, a single probability distribution is required in order to ensure a rational decision (Lindley 1971). Such a probability distribution is supposed to reflect beliefs and is elicited as such from experts. On the contrary, the use of imprecise probabilities is supposed to reflect the actual objective information collected about a given risky process. Hence, from a Bayesian point of view, there is a gap between results provided by joint uncertainty propagation methods and the expected scientific judgment a risk analysis procedure should lead to (Aven 2010).

In this paper, we outline a complete risk-analysis methodology that maintains the difference between aleatory and epistemic uncertainties throughout the process, and propose a knowledge elicitation strategy to that effect. After proposing a unified outlook of modern uncertainty theories in Section 2, a general uncertainty elicitation methodology is outlined in section 3, whose main message is to adapt the choice of the representation tool to the richness of the available information. Basic joint uncertainty propagation techniques are then described in Section 4. In Section 5, we propose a subjective approach to circumvent the difficult issue 
of deciding under incomplete information. The idea is to re-introduce the decision-maker's subjectivity, in the style of Hurwicz criterion, by means of an optimism coefficient. This is done at the final decision-making stage, rather than at the uncertainty elicitation stage as is often the case with the Bayesian approach. Finally, an example of health risk calculation is presented in Section 6, as an illustration of the proposed decision methodology.

\section{THEORIES OF UNCERTAINTY TOLERATING INCOMPLETENESS}

There are basically three mathematical frameworks for the joint modelling of aleatory and epistemic uncertainty: convex probability sets, random sets and possibility theory (Dubois and Prade 2009). Dempster (1967) was among the first scholars to suggest articulating probability theory with a faithful representation of incomplete information, replacing a random variable by a multiple-valued mapping describing limited knowledge on the actual values it takes. Upper and lower probability bounds for events on the range of the random variable are then obtained. Shafer (1976) later interpreted these bounds as subjective plausibility and belief functions induced by incomplete unreliable evidence. The idea is to assign subjective probability weights to sets of possible values, instead of point values (as in classical probability functions). This random set formalism (Kendall 1974) thus allows a common framework for representing both types of uncertainty (epistemic and stochastic). In the socalled possibility theory (Zadeh 1978, Dubois and Prade 1988) fuzzy set membership functions are used as primitive entities for representing incomplete information. Information items are then viewed as nested sets of possible values, which is particularly suitable for representing human-originated incomplete/imprecise information (Dubois 2006). It can be viewed as a computationally simple special case of the previous formalism, restricted to nested sets. Walley (1991) developed a more general imprecise probability theory whereby the issue of partial lack of probabilistic information is addressed by means of convex sets of probability functions. These convex sets can be used to represent incomplete information about a probabilistic model as in robust statistics (Huber 1981), or (this is Walley's stance) as subjective uncertainty where lower expectations are interpreted as maximal buying prices for gambles. 


\subsection{Imprecise probability}

Basically, an objective probabilistic representation is incomplete if a family of probability functions $\mathcal{P}$ is used in place of a single distribution $\mathrm{P}$, because the available information is not sufficient for selecting a single one in $\mathcal{P}$. Under such imperfect knowledge it is only possible to compute optimal bounds on the probability of measurable events $A \subseteq S$ :

$$
P^{*}(A)=\sup \{P(A) P \in \mathcal{P}\}, P *(A)=\inf \{P(A) P \in \mathcal{P}\}
$$

The upper bound $P^{*}(A)$ can be used to measure the degree of plausibility of $A$, evaluating to what extent $A$ is not impossible, i.e., there is no reason against the occurrence of $A$. The lower bound $P *(A)$ can be used to measure the degree of certainty of $A$. This is similar to the standard probabilistic framework where the degree of belief in an event is equated to its frequency of occurrence if the latter is available (this is the Hacking principle).

It is obvious that $P *(A)=1-P^{*}\left(A^{c}\right)$, where $A^{c}$ is the opposite event of $A$. It expresses the idea that an event $A$ is certain if and only if its opposite is impossible. Each event $A$ is then assigned an interval $\left[P *(A), P^{*}(A)\right]$, which is all the larger as information is lacking. In the face of ignorance, the consistent representation consists of using the trivial bounds $[0,1]$. Reasoning with such bounds is generally not equivalent to using the set $\mathcal{P}$ because in general the set of probability functions respecting the bounds $\{P, P \geq P *\}=\left\{P, P \leq P^{*}\right\}$ is convex and strictly contains $\mathcal{P}$ (the notation $P \geq P *$ is short for $\forall A \subseteq S, P(A) \geq P *(A)$ ).

Conversely, imprecise probabilistic information may take the form of lower probability bounds $P_{-}\left(A_{i}\right)$ of specific events $\left\{A_{i}, i=1, \ldots k\right\}$. The value $P_{-}\left(A_{i}\right)$ can be understood either as a lower bound of the frequency of occurrence of $A_{i}$, as known by an agent, or as the subjective belief of this agent about the occurrence of $A_{i}$. In the latter case, belief is measured as the greatest buying price of a lottery ticket that some decision-maker accepts to pay in order to win $1 \$$ if $A_{\mathrm{i}}$ occurs (Walley 1991). In order to make sense, these bounds must be such that the set $\mathcal{P}=\left\{P, P\left(A_{i}\right) \geq P_{-}\left(A_{i}\right), i=1, \ldots k\right\}$ is not empty (which is the no sure loss condition of Walley 1991). They must be optimal in the sense that best lower bounds $P *\left(A_{i}\right)$ (as obtained from $\mathcal{P}$ via Eq. 1) should coincide with assessments $P_{-}\left(A_{i}\right)$ for all $i=1, \ldots k$ (there is no point in buying the lottery tickets more than $\left.P_{-}\left(A_{i}\right)\right)$. More generally, a lower envelope function $P_{-}$is said to be coherent (according to Walley) if:

$$
P *(A)=\inf \left\{P(A), P \geq P_{-}\right\}=P_{-}(A), \forall A \subseteq S
$$

Note that this model of subjective belief is similar to subjective probability, but it differs from it on a basic issue: in the classical theory, $P *(A)$ is also the least selling price of the 
lottery ticket pertaining to the occurrence of event $A$. Here this selling price is just requested to be not less than $P *(A)$ and it coincides with $P^{*}(A)=1-P *\left(A^{c}\right)$. The interval $\left[P *(A), P^{*}(A)\right]$ represents the amount of ignorance of the agent, i.e. to what extent this agent is reluctant to engage into a fair betting process (or a full-fledged probabilistic belief assessment). Like subjective probabilities, values $P_{-}\left(A_{i}\right)$ can be elicited; unlike subjective probabilities, they tolerate some amount of ignorance to be expressed.

\subsection{Possibility theory and set-valued representations}

An extreme example of such a representation is when all that is known is that some parameter $x$ taking value on the space $S$ is only known to belong to a subset $E$ of $S$. Note that the set $E$ is made of mutually exclusive elements, since each realization of $x$ is unique. $E$ is said to be a disjunctive set and represents an epistemic state. Then, Boolean plausibility and certainty functions can be respectively defined by:

$$
\begin{aligned}
& \Pi(A)=1 \text { if } A \cap E \neq \varnothing, \text { and } 0 \text { otherwise; } \\
& N(A)=1-\Pi\left(A^{c}\right)=1 \text { if } E \subseteq A \text { and } 0 \text { otherwise. }
\end{aligned}
$$

Clearly $N(A)=1$ if and only if $x \in A$ is implied by the available information (hence its certainty) while $\Pi(A)=1$ if and only if $x \in A$ is consistent with the available information. The associated probability set contains all probability functions with support inside set $E: P=\{P$, $P(E)=1\}$. Note that the statement “ $\mathrm{x} \in E$, " represents subjective information about $x$. In the Bayesian framework, a probability distribution on $E$ should be assigned. Using a mere set indicates that the agent refuses to buy lottery tickets pertaining to events $A$ not implied by $E$ (he assigns $P_{-}(A)=0$ to those events).

A refined situation is when some elements in $E$ are considered to be more plausible than others for $x$ and degrees of possibility $\pi(r) \in[0,1]$ can be assigned to $r \in E$, with condition that $\pi(r)=0$ if $r \notin E$ and $\pi(r)=1$ for at least one value $r \in E$. Plausibility and certainty functions can be respectively defined by means of so-called possibility and necessity measures (Dubois and Prade 1988) generalizing the above Boolean functions:

$$
\Pi(A)=\sup _{r \in A} \pi(r) ; N(A)=1-\Pi\left(A^{c}\right)=i n f_{r \notin A} 1-\pi(r)
$$

Interestingly, necessity functions are coherent in the sense of Walley, so that $\Pi$ and $N$ define a family of probability measures $\mathcal{P}(\pi)=\{P, P \geq N\}$ such that $\Pi=P^{*}$ and $N=P *$, where $P *(A)=\inf \{P(A), P \geq N\}$ (Dubois and Prade 1992). 


\subsection{Random disjunctive sets}

It is also possible to assign reliability weights $m\left(E_{i}\right)$ to statements of the form " $x \in E_{i}$ ", whereby $m\left(E_{i}\right)$ expresses the probability that the statement " $x \in E_{i}$ " accurately represents the available information (Shafer 1976). It is a de dicto probability, not to be confused with the de re probability of the occurrence of event $E_{i}$. Assuming a number $k$ of such statements each having probability $m\left(E_{i}\right)$, this approach comes down to considering a probability distribution $m$ over the family of subsets $E$ of $S$, such that $m(\varnothing)=0$, and $\sum_{E \subseteq S} m(E)=1$. This is what is usually called a random set ${ }^{1}$. The weight $m(E)$ is the amount of probability that could be assigned to elements in $E$, but is not by lack of information. This is the randomized version of the plain incomplete information case " $x \in E$ " (where then, $m(E)=1$ ). Total ignorance is then when $m(S)=1$. So called belief and plausibility functions are defined as:

$$
\operatorname{Bel}(A)=\sum_{E \subseteq A} m(E) ; \operatorname{Pl}(A)=1-\operatorname{Bel}(A)^{c}=\sum_{E \cap A \neq \emptyset} m(E)
$$

They obviously generalize Boolean functions in (3) and (4). They also generalize necessity and possibility measures in (5). The latter are obtained in the case of consonance, when the set $\mathcal{F}=\{E, m(E)>0\}$ of focal subsets is nested, i.e. $\forall E, E^{\prime} \in \mathcal{F}, E \subseteq E^{\prime}$ or $E^{\prime} \subseteq E$. Then $P=P l$ and $N=B e l$, and the possibility distribution $\pi$ is such that $\pi(r)=P l(\{r\})$. A unique probability function $P$ is retrieved if all focal sets are singletons; then the mass function $m$ is a probability distribution and $\mathrm{Bel}=\mathrm{Pl}=\mathrm{P}$. Belief functions are coherent lower envelopes that exactly encode the convex set of probability measures $\mathcal{P}(m)=\{P, P \geq B e l\}$. A typical (consonant) case of a belief function is an unreliable testimony of the form " $x \in E$ " where there is some probability $p$ that the information is irrelevant. It defines a mass function such that $m(E)=1-$ $p$ and $m(S)=p$ (when the information is irrelevant, it is useless). It comes down to a piece of information of the form $P(E) \geq 1-p$, i.e. a confidence set. Note that the mass function $m$ can have a frequentist flavor $(m(E)$ is then the frequency of imprecise outcomes of the form $E$ ) or a subjective flavor $(m(E)$ is then the assigned subjective probability that $E$ is the correct information).

\footnotetext{
1 A random disjunctive set, in fact. There is a branch of random set theory (Kendall, 1974) where the set-valued realizations represent objective entities (e.g. a shape to be located in some area). In contrast, in this paper, set-valued realizations are epistemic constructs representing incomplete information.
} 


\section{TOWARD FAITHFUL REPRESENTATIONS OF UNCERTAINTY}

Possibly one of the most important reasons why alternative methods are needed for representing uncertainties in environmental risk assessments is the quest for consistency with available information. When an investigator, faced with incomplete/imprecise information, decides to overlook this partial lack of knowledge and resorts to postulating a unique subjective probability distribution function (PDF), he/she is arguably misrepresenting the available information. Indeed, there is then no formal difference between known stochastic variability and incomplete information as soon as objective and subjective probability distributions are jointly propagated.

\subsection{Practical representations}

Whilst known variability can be captured by precise probability distributions, we propose to use intervals and representations that refine them for consistently representing partial ignorance. Figure 1 is a flowchart that proposes to choose specific mathematical representations of information pertaining to a model parameter, according to the actually available information regarding this parameter, thus offering an elicitation strategy. The following simple uncertainty representations based on intervals or their generalisation are used in the flowchart:

- $\quad$ an interval $[a, b]$, such that the value of the parameter $\mathrm{x}$ under concern is supposed to lie in it.

- a fuzzy interval (Dubois and Prade 1988), defined by a possibility distribution $\pi: \boldsymbol{R} \rightarrow$ $[0,1]$, that assigns to each value $r$ of $x$ a degree of possibility $\pi(r) \in[0,1]$. It is a generalized interval insofar as $\forall \lambda \in(0,1]$, the cut set $I_{\lambda}=\{x, \pi(x) \geq \lambda\}$ is a closed interval, and the core $I_{l}$ is not empty.

- a p-box defined by a pair of (cumulative) probability distribution functions (PDF) $\left(F^{*}\right.$, $F_{*}$ ) where $F^{*}>F_{*}$. It characterizes a family $\mathcal{P}_{\text {pbox }}$ of probability functions with PDFs $F$ such that $F^{*} \geq F \geq F_{*}$ (Williamson and Downs 1990).

All of these representations correspond to special kinds of random intervals in the style of Dempster-Shafer. Namely, a fuzzy interval can be viewed as a multiple-valued mapping from $[0,1]$, equipped with the Lebesgue measure, to intervals, assigning a focal set $I_{\lambda}$ to each $\lambda \in$ $(0,1]$. A fuzzy interval can also be viewed as a probability family. More precisely, each set $I_{\lambda}$ can be viewed as a confidence interval containing the value of $x$ with confidence at least $1-\lambda$. That is, the possibility distribution $\pi$ encodes the probability family: 


$$
\mathcal{P}(\pi)=\left\{P, P\left(I_{\lambda}\right) \geq 1-\lambda, \forall \lambda \in(0,1]\right\} .
$$

As said above, the possibility measure $\Pi$ induced by $\pi$ on events A satisfies:

$$
\Pi(A)=\sup _{r \in A} \pi(r)=\sup \{P(A) P \in \mathcal{P}(\pi)\} .
$$

Very common in probability are inequalities of the form $P\left(I_{\lambda}\right) \geq 1-\lambda, \forall \lambda \in(0,1]$. For instance Chebyshev inequality reads $P\left(X \in\left[x^{\text {mean }}-x, x^{\text {mean }}+x\right]\right) \geq 1-\sigma^{2} / x^{2}$, for $x \geq \sigma$, where $x^{\text {mean }}$ is the mean and $\sigma$ a standard deviation. Intervals of the form $\left[x^{\text {mean }}-x, x^{\text {mean }}+x\right]$ define a fuzzy interval with core $\left[x^{\text {mean }}-\sigma, x^{\text {mean }}+\sigma\right]$ and infinite support. This possibility distribution encodes a family containing all probability distributions with mean $x^{\text {mean }}$ and standard deviation $\sigma$. Likewise the triangular fuzzy interval with support $[a, b]$ and mode $c$ encodes a family containing all probability distributions with such mode and support lying in $[a, b]$ (Baudrit and Dubois 2006).

Another popular example of a set-valued probabilistic representation is a probability box (Ferson et al. 2003). A p-box is also a random interval, replacing the above intervals $I_{\lambda}$ by other intervals of the form $\left[F^{*-1}(\lambda), F_{*}^{-1}(\lambda)\right]$ playing the role of focal sets (Kriegler and Held 2005, Destercke et al. 2008). It is possible to extract a p-box from a fuzzy interval, letting $F^{*}(r)=\Pi(x \leq r)$ and $F *(r)=N(x \leq r)$. However, this is a special p-box such that $F^{*}(r)=1$ and $F_{*}(r)=0$ for some value $r \in \boldsymbol{R}$ (namely take $r$ such that $\pi(r)=1$ ). In other words, this p-box contains a Dirac function. However this p-box contains less information than $\pi$ because the probability set $\mathcal{P}(\pi)$ is strictly included in the probability family $\mathcal{P}_{\text {pbox }}$ induced by this p-box (Baudrit and Dubois 2006).

Similarly, a p-box can be extracted from a random interval inducing belief and plausibility functions, considering $F^{*}(r)=P l(x \leq r)$ and $F_{*}(r)=\operatorname{Bel}(x \leq r)$. Again, this p-box is less informative than the random interval it is built from, and it is equivalent to another belief function. Indeed a random interval $\left\{\left(\left[a_{i}, b_{i}\right], m_{i}\right), i=1, \ldots, n\right\}$ is equivalent to a p-box (i.e. they yield the same probability bounds for all events) if and only if the ordering of the lower bounds of intervals $\left[a_{i}, b_{i}\right]$ is the same as the ordering of the upper bounds: $a_{i} \leq a_{j}$ if and only if $b_{i} \leq b_{j}$. For instance, a set of measurements $\left\{r_{i}, i=1, \ldots, k\right\}$ with fixed error $e$, corresponding to focal intervals $\left[r_{i}-e, r_{i}+e\right]$ yields a belief function that coincides with a p-box. More general practical representation techniques are discussed and related to each other in Destercke et al. (2008). 


\subsection{An elicitation methodology}

The input point to the flowchart in Figure 1 considers whether or not the investigator wishes to represent a given risk model parameter by a deterministic quantity (i.e., not subject to variability). There may be several reasons for assuming a parameter should take on a fixed value. For example, the investigator may know that the value of the parameter is indeed a constant (e.g. the height of a chimney stack or the depth of a well); on the contrary he may know that he will never get information regarding the parameter's variability (whether spatial or temporal); therefore he chooses to assume a constant value, albeit imprecisely known. Once the user of the flowchart has chosen whether he wishes to use a constant parameter value or not, he is guided through a series of questions that assist him in selecting an appropriate tool for representing the information available to him.

If a representation by a constant parameter is selected, questions are asked in order to identify the degree of precision of this information regarding the parameter value. Questions go from the less to the more informed. First the user is asked whether he can identify an interval that contains the parameter value with certainty. If this is the only information that can be provided, then a simple interval $[a, b]$ will be assigned to the parameter. If the user can express preferences within this interval, it can be refined into a trapezoidal or a triangular fuzzy set. In fact, an interval $[a, b]$ and a plausible value $r *$ therein (interpreted as the core of the fuzzy interval: $\pi\left(r^{*}\right)=1$ ) can be modelled as a triangular fuzzy interval. The corresponding probability set $\mathcal{P}(\pi)$ then contains, among other ones, all probability functions with unimodal density with support in $[a, b]$ and mode $r^{*}$ (Baudrit and Dubois 2006). Instead of a plausible value, an interval thereof can be used as a core of a trapezoidal fuzzy interval. If the available information is not sufficient for defining a sensible interval containing some parameter, one may resort to considering a set of representative scenarios where assumptions can be stated, each leading to a sensible interval.

If the user decides to consider the parameter as a random variable, he is asked whether statistical data are available regarding the parameter and, if so, whether there are a sufficient number of precise measurements. At this stage a distinction must be made according to whether the variability is spatial or temporal. In the case of spatial variability and if sufficient data are available, geostatistics models (e.g. Chilès and Delfiner 1999) can be used to represent the spatial variability and provide reliable estimators of the parameter. In the case of temporal variability and of sufficient available data, a unique probability distribution will be the appropriate representation tool. If there is a large number of imprecise data, a random set 
can be used. But if only a little amount of data is available, and one must basically rely on expert knowledge, then the investigator is asked whether he can provide the support of the distribution describing the parameter's variability. Should this be the case, if the user knows what type of distribution is suitable and can provide, based on expert knowledge, intervals for the parameters of the distribution (e.g. average and standard deviation), then a parametric probability family (represented as a p-box) can be used. Note however that the use of a p-box may represent a certain loss of information as the latter represents a non-parametric family of PDFs even if bounded by parametric ones (see Baudrit et al. 2008 for a discussion and a proposal for handling such imprecise parametric models). If knowledge on the distributions is not available, the flowchart resorts to the fuzzy interval-type representations mentioned previously, now supposed to represent a family of objective probabilities. In the case of imprecise geostatistical data, specific techniques can be used, for instance, the pioneering fuzzy interval approach of Bardossy et al. (1988) (see Loquin and Dubois 2010, for a survey)

The list of tools in the flowchart, which is by no means exhaustive, is drawn from the uncertainty theories cited in the previous section and attempts to cover the variety of "degrees of precision” typically encountered in the field of environmental risks. While inherently incomplete, the main benefit of the proposed flowchart is to bring the user to realize that there is no one-all-fit-all method for representing uncertainty. All depends on the nature of the available information. Once appropriate representations have been selected for all uncertain risk model parameters, the information can be propagated using the techniques recalled in the next section, the choice of which depends not only on the information representation tools, but also on possible dependencies between model parameters.

\section{UNCERTAINTY PROPAGATION}

Already in the 1980's Kaufmann and Gupta (1985) had proposed so-called "hybrid numbers” which simultaneously express imprecision and randomness. Later on, Cooper et al. (1996) used this framework to combine stochastic and subjective sources of data uncertainty in the estimation of risk. More recently, Guyonnet et al. (2003) use probability distributions for representing variability in model inputs, and fuzzy intervals when only partial information is available on other inputs. They proposed a propagation method, also termed "hybrid", combining Monte Carlo sampling of probability distributions with fuzzy interval analysis (Dubois et al. 2000), thus generating a random fuzzy interval as the system output. Baudrit et al. (2005) identified a consistent approach for summarizing the results of this method, in the 
form of a probability box (closely related to Dempster upper and lower probabilities, and belief functions of Shafer). Baudrit et al. (2006, 2007) proposed an alternative uncertainty propagation method, called the independent random set (IRS) method, where the random sampling procedure is applied not only to the probability distributions, but also to the fuzzy intervals. Couso et al. (2000) showed that this method is a conservative counterpart to the calculation with random quantities under stochastic independence (classical Monte Carlo method). Baudrit et al. (2007) showed that the IRS method yields very similar results to those of the hybrid method, differences being due to different hypotheses with respect to model parameter dependencies (Baudrit et al. 2006). Other authors directly model incomplete information by probability boxes and provide suitable uncertainty propagation methods (Williamson and Downs 1990; Regan et al. 2004).

We recall two propagation methods, one of which will be illustrated in section 5 . They are well suited for situations involving both epistemic and stochastic uncertainty. The first method (Guyonnet et al. 2003) combines the random sampling of probability distribution functions (PDFs) with interval analysis on the cut sets of fuzzy intervals. We consider a generic risk model that is a function of a certain number of parameters $x_{1}, \ldots x_{n}, y_{1}, \ldots y_{m}$ :

$$
z=f\left(x_{1}, \ldots x_{n}, y_{1}, \ldots y_{m}\right)
$$

where $z$ is risk model output; $x_{1}, \ldots x_{n}$ are $n$ independent model parameters represented by

probability distribution functions (PDFs) $F_{1}, \ldots, F_{\mathrm{n}} ; y_{1}, \ldots y_{m}$ are $m$ model parameters represented by fuzzy intervals with possibility distributions $\pi_{1}, \ldots, \pi_{\mathrm{m}}$.

\subsection{A fuzzy Monte-Carlo method}

The so-called "hybrid" procedure of Guyonnet et al. (2003) is as follows:

1. Generate $n$ random numbers $\left(\chi_{1}, \ldots, \chi_{n}\right)$ in $[0,1]^{n}$ from a uniform distribution and sample the $n$ PDF's to obtain a realization of the $n$ random variables: $r_{1}, \ldots r_{\mathrm{n}}$, where $r_{i}$ $=F^{-1}\left(\chi_{i}\right)$.

2. Select a possibility value $\lambda$ in $[0,1]$ and build cut-sets of $\pi_{1}, \ldots, \pi_{\mathrm{m}}$ at level $\lambda$ yielding intervals $I_{j \lambda}=\left\{r, \pi_{j}(r) \geq \lambda\right\}$.

3. Interval calculation: calculate the Inf (least) and $\operatorname{Sup}$ (greatest) values of interval $Z=$ $f\left(r_{1}, \ldots, r_{\mathrm{n}}, I_{1 \lambda}, \ldots, I_{m \lambda}\right)$, scanning all values located within the cut sets of each fuzzy set. 
4. Consider these Inf and Sup values to be the lower and upper limits of the cut set containing the output $z$ at possibility level $\lambda$.

5. Return to step 2 and repeat steps 3 and 4 for another value of $\lambda$. The fuzzy result describing $z$ is obtained from the Inf and Sup values of $Z$ for each cut set.

6. Return to step 1 to generate a new realization of the random variables.

Note that step 3 may need a stochastic search method if the function $f$ is not monotonic and its extrema are ill-known. Computations can be arranged so as to avoid redoing them for each $\lambda$-cut (for instance using the transformation method of Hanss 2004).

A family of $\omega$ possibility distributions (a random fuzzy set) is thus obtained that describe the output value $z$ ( $\omega$ being the number of realizations of the random variables). This random fuzzy set can be interpreted as a standard random interval as proposed by Baudrit et al. (2005), namely separately collecting all intervals of the form $f\left(r_{1}, \ldots, r_{\mathrm{n}}, I_{1 \lambda}, \ldots, I_{m \lambda}\right)$ for all samples $\left(\chi_{1}, \ldots, \chi_{n}, \lambda\right)$.

\subsection{The independent random set approach}

An alternative propagation method is based on independent random sets (called IRS; Baudrit et al. 2006). It exploits the fact that the theory of evidence (Shafer 1976) encompasses both possibility and probability theory. It is based on an extension of the Monte-Carlo scheme whereby sampling is performed likewise on random variables, on possibility distributions $\pi_{1}$, ..., $\pi_{\mathrm{m}}$ (and p-boxes, if any input parameter representation takes such a form) associated to each imprecise parameter. The procedure is as follows:

1. Generate $n+m$ random numbers $\left(\chi_{1}, \ldots, \chi_{n+m}\right)$ in $[0,1]^{n+m}$ from a uniform distribution on $[0,1]$.

2. Sample the $n$ PDF's to obtain a realization of the $n$ random variables: $r_{1}, \ldots r_{\mathrm{n}}$,

3. Sample the $m$ fuzzy intervals $\pi_{1}, \ldots, \pi_{\mathrm{m}}$ (or p-boxes) each at a different level $\chi_{n+i}$ to obtain $m$ intervals: $I_{1}, \ldots, I_{\mathrm{m}}$, where $I_{i}=\left\{r, \pi_{i}(r) \geq \chi_{n+i}\right\}$.

4. Calculate the $\operatorname{Inf}$ (least) and $\operatorname{Sup}$ (greatest) values of $z=f\left(p_{1}, \ldots, p_{\mathrm{n}}, I_{1}, \ldots, I_{\mathrm{m}}\right)$, using interval analysis, considering all values located within the intervals $I_{1}, \ldots, I_{\mathrm{m}}$.

5. Return to step 1 to generate a new realization of the random variables and the fuzzy sets (or p-boxes). Repeat $\omega$ times. 
Again, a random interval is obtained. The difference between the "hybrid" and IRS schemes lies in the assumptions with respect to independence between model parameters. In the "hybrid" scheme, stochastic independence between the probabilistic variables is often assumed, although non-linear monotone dependency between the random variables can be accounted for by means of rank correlation methods (Connover and Iman 1982). Stochastic independence between the group of probabilistic variables and the group of possibilistic quantities is also assumed. But the fuzzy interval analysis in the "hybrid" scheme assumes that the sources supplying information related to imprecise parameters are totally dependent, while no link between the parameters themselves is assumed. In contrast with the second IRS Monte-Carlo scheme, the "hybrid" method comes down to restricting the samples $\left(\chi_{1}, \ldots\right.$, $\left.\chi_{\mathrm{n}+\mathrm{m}}\right)$ to those of the form $\left(\chi_{1}, \ldots, \chi_{\mathrm{n}}, \lambda \ldots, \lambda\right)$. On the other hand, the IRS method assumes independence between all information sources.

\subsection{Presentation of the results}

The output random interval is then summarized in the form of a pair of upper and lower cumulative probability distributions, i.e. a p-box (Baudrit et al. 2005), considering $F^{*}(\theta)=P l(x$ $\leq \theta)$ and $F_{*}(\theta)=\operatorname{Bel}(x \leq \theta)$, as per the theory of evidence recalled above. It evaluates the probability of the proposal $x \leq \theta$, i.e., "the calculated risk lies below a specified target level $\theta$ '. The probability that this proposal is true is comprised between the degree of plausibility (an upper bound on probability) and the degree of belief (a lower bound on probability). Therefore, the lower bound $\operatorname{Bel}(x \leq \theta)$ gathers the imprecise evidence that asserts $x \leq \theta$ while the upper bound $P l(x \leq \theta)$ gathers the imprecise evidence that does not contradict $x \leq \theta$. The interval $[\operatorname{Bel}(x \leq \theta), P l(x \leq \theta)]$ contains all potential probability values compatible with the mass function $m$ obtained from the propagation step.

The significant advantage of standard probabilistic methods using the Monte Carlo method with arbitrarily selected PDFs despite incomplete/imprecise information is that a single value for the probability of exceeding the critical threshold $\theta$ is obtained. This will appear more appealing to decision-makers dealing with environmental risks than imprecise probabilities of exceeding such a threshold. In fact, Bayesian scholars deny the potential of approaches like the above one to provide useful support for obtaining a scientific judgment about the unknown quantities under concern, considering that the explicit handling of ignorance on top of available statistical probabilities only leads to an objective description of these unknown quantities (Aven 2010). However it can be argued that the probability bounds at work in these 
representations are subjective, whether or not an objective probability rules the behaviour of the unknown quantities: from one source or expert to another, the probability bounds will be different without necessarily being conflicting (Dubois 2010).

In order to increase the acceptance of methods that account for epistemic uncertainties in the field of environmental or health risks, it is proposed to introduce an additional reasoning step in order to provide a result that is more amenable to potential users.

\section{A HURWICZ STYLE APPROACH TO DECISION UNDER PARTIAL IGNORANCE}

The classical approach to decision under uncertainty is due to Savage (1954). Decision under epistemic uncertainty is there opposed to "decision under risk", where the latter presupposes the knowledge of precise objective probabilities of occurrence of states of nature, a situation that is not met in our setting. Even when such probabilities are ill-known, Savage has suggested that, provided some postulates of rational decision are accepted, a decisionmaker should make decisions as if he had a unique subjective probability distribution in mind when ranking potential decisions, the ranking being done according to the expected utility criterion. This view has been challenged to a large extent for at least two reasons: first the expected utility criterion neglects the attractiveness of sure gains against lotteries which may have higher expectations but where greater losses are possible as well. Second, in the face of partial ignorance decision makers may fail to use the same subjective probability in successive choices when comparing decisions in a pairwise manner (Ellsberg paradox; Ellsberg 1961).

\subsection{Decision Under Partial Ignorance}

Under epistemic uncertainty, the result of the risk analysis, as we described it, consists in a random set, typically having the form of a probability box, i.e., a pair of PDFs $\left(F^{*}, F_{*}\right)$ where $F^{*}>F_{*}$. As pointed out above, it corresponds to a uniform mass density assigned to subsets of the form:

$$
A_{\lambda}=\left[F^{*-1}(\lambda), F^{-1}(\lambda)\right] \text { where } F^{-1}(\lambda)=\inf \{x, F(x) \geq \lambda\}
$$

In the case of discrete PDFs, as typically obtained from our algorithms, it comes down to a finite set of $n$ intervals $\left[a_{i}, b_{i}\right]$ each being assigned a probability weight $m_{i}$, which represents the proportion of results of the form $\left[a_{i}, b_{i}\right]$, obtained by the joint Monte-Carlo/interval analysis method. Comparing decisions when the uncertainty is described by a random set is 
problematic because expected utilities will take the form of intervals, and intervals are not totally ordered. Likewise, probabilities of relevant events will be only known via a probability interval.

A number of decision criteria have been proposed in the literature, both in economics (see Chateauneuf and Cohen 2009 for a survey) and in connection with Walley's imprecise probability theory, following pioneering works by Isaac Levi (see Troffaes 2007). There are basically three schools of thought:

- Comparing set-valued utility estimations under more or less strict conditions. These decision rules, such as Levi's E-admissibility usually do not result in a total ordering of decisions, and some scholars may consider that the problem is not fully solved then. Nevertheless they provide rationality constraints on the final decision.

- Comparing point-valued estimations after selection of a « reasonable » utility value within the computed bounds. For instance the generalisation of the (pessimistic) maximin criterion of Wald proposed by Gilboa and Schmeidler (1989).

- Selecting a probability measure in the set of imprecise priors and ranking decisions following the corresponding expected utility. This is the approach proposed by Smets (2005) with his so-called pignistic probability.

The two latter approaches lead to clear-cut best decisions but the responsibility of the choice of the point-valued risk measure then relies on the decision-maker. In the second approach, the choice of the equivalent subjective probability depends on the pair of decisions to be compared, while in the third approach, the subjective probability function is chosen once and for all.

\subsection{The confidence index}

In our setting, deciding if the output of the system under study lies beyond a critical threshold $\theta$ may be difficult: we have to compare the ill-known expected value:

$$
E V=\left[\sum_{i=1, \ldots, n} m_{i} a_{i}, \sum_{i=1, \ldots, n} m_{i} b_{i}\right]
$$

to the threshold $\theta$, or to compute an imprecise probability of violating it:

$$
I P=\left[1-F^{*}(\theta), 1-F *(\theta)\right] .
$$

Such an interval may baffle a decision-maker, if too wide. An important characteristic of the field of environmental and health risks is that public perception is one of "aversion to risk". Obviously, in such a context, it would not be acceptable to use the optimistic bound $1-$ $F^{*}(\theta)$ on probability as the sole indicator of the acceptability of risk. Note that the optimistic bound will be the $\mathrm{Bel}$ indicator, if the event $B$ whose likelihood is to be judged is that a risk 
threshold $\theta$ is exceeded ( " $x>\theta$ "), and $P l\left(P l\left(B^{c}\right)=1-\operatorname{Bel}(B)\right.$ ), if the event is $B^{c}$, i.e, that risk lies below the threshold $\theta$. One might then consider that the pessimistic bound on probability should be used as the unique indicator of acceptability. This approach, while being conservative, presents the important disadvantage of ignoring all the information leading to less pessimistic estimates of risk.

Insofar as a decision has to be selected, the lesson of Savage theory is that a probability function $P \in \mathcal{P}$ must be selected so as to account for the final ranking of decisions. In the case when expected utility is the ranking criterion, it enables the selection of a unique probability value $p \in I P$ of violating the critical threshold $\theta$, or a single expected value within the interval $E V$. These probability and expected value blend the objective available information (inducing the interval IP) and the attitude of the decision-maker in front of partial ignorance.

There are three approaches for selecting a single probability function in a family thereof:

1. Applying the Laplace principle of insufficient reason to each focal set $\left[a_{i}, b_{i}\right]$, thus changing it into a uniformly distributed PDF $F_{i}$ on $\left[a_{i}, b_{i}\right]$, and using the distribution function $F_{1}=\sum_{i}=1, \ldots, n m_{i} F_{i}$, to compute an expected value $E V_{1}$ and a violation probability $p_{1}=1-F_{1}(\theta)$.

2. Replacing each focal set $\left[a_{i}, b_{i}\right]$ with a value $f\left(a_{i}, b_{i}\right) \in\left[a_{i}, b_{i}\right]$, where $f$ is increasing in both places; then using the distribution function $F_{2}$ induced by the probability assignment $\left\{\left(f\left(a_{i}, b_{i}\right), m_{i}\right), i=1, \ldots, n\right\}$. This PDF $F_{2}$ has pseudo-inverse $F_{2}^{-1}(\lambda)=$ $\inf \left\{x, F_{2}(x) \geq \lambda\right\}=f\left(a_{i}, b_{i}\right), \forall \lambda \in[0,1]$, where $A_{\lambda}=\left[a_{i}, b_{i}\right]$. Then the expected value $E V_{2}$ takes the form of $E V_{2}=\sum_{i=1, \ldots, n} m_{i} f\left(a_{i}, b_{i}\right)$.

3. Directly selecting a PDF $F_{3}$ such that $F_{3}(x)=g\left(F^{*}(x), F_{*}(x)\right) \in\left[F^{*}(x), F_{*}(x)\right]$.

The first method was proposed by Smets (2005) under the name "pignistic transformation" and axiomatically justified. It is identical to the so-called Shapley value used in cooperative game theory as a fairness principle for sharing benefits across members of coalitions. Beyond its formal appeal, its drawback in our context is that it leaves no room to a decision-maker for expressing his attitude in front of risk. The pignistic transformation just explains how an individual is likely to bet in the face of ignorance if he is forced to bet: namely using a twostepped procedure:

- $\quad$ Bet on $\left[a_{i}, b_{i}\right]$ with subjective probability $m_{i}$;

- then bet uniformly on some value within $\left[a_{i}, b_{i}\right]$, as there is no reason to favor one value against another. 
The second method was advocated by Jaffray $(1988,1994)$. Its aim is to try and preserve the linearity of the expected utility. The idea is to assign a preference relation on belief functions on states of nature in place of a set of probability distributions (lotteries), and apply the axioms of decision under risk to a functional that, to any belief function $\mathrm{Bel}$, assigns a precise expected value $E V_{B e l}$, still obeying the famous independence and continuity axioms of decision theory (after Herstein and Milnor 1953), which ensure the linearity of the expected utility, i.e.:

$$
E V_{a B e l+(1-a) B e l}=a E V_{B e l}+(1-a) E V_{B e l},
$$

In fact this approach is the same as the traditional Von Neumann and Morgenstern (1947) approach to decision under risk, where epistemic uncertainty is accounted for by replacing the set of states of nature by the set of epistemic states of the decision maker, each possible epistemic state being modeled by a set of states of nature, one of which is the right one. Jaffray considers a belief function as an objective probability over epistemic states. The quantity $f\left(\left[a_{i}, b_{i}\right]\right)$ is then like the equivalent (subjectively perceived) risk level of the epistemic state $\left[a_{i}, b_{i}\right]$.

Moreover he adds a further dominance axiom enforcing the monotonic increasingness of $f$ (hence $\mathrm{EV}_{\mathrm{Bel}}$ ) with respect to the following partial ordering between intervals (viewed as random sets with mass assignment 1 ):

Dominance axiom: $\left[a_{i}, b_{i}\right] \geq\left[a_{j}, b_{j}\right]$ if and only if $a_{i} \geq a_{j}$ and $b_{i} \geq b_{j}$.

Then he proves that $f\left(\left[a_{i}, b_{i}\right]\right)$ is of the form $f\left(a_{i}, b_{i}\right)$ and $E V_{B e l}$ is of the form of criterion $E V_{2}=\sum_{i=1, \ldots, n} m_{i} f\left(a_{i}, b_{i}\right)$ above, that is, the precise expectation only depends on the value of the end-points of focal intervals. Interestingly, the pignistic transformation is also linear with respect to the convex combination of belief functions. The difference is that the choice of a value $f\left(a_{i}, b_{i}\right)$ to which weight $m_{i}$ is assigned (in method 2) is replaced by a uniformly distributed probability in Smets' method. To make the latter more flexible, one could as well use any probability measure on $\left[a_{i}, b_{i}\right]$, that reflects the attitude of the decision-maker when the latter only knows that the real value of the parameter (e.g. pollution index) lies between $a_{i}$ and $b_{i}$ (in agreement with the Bayesian approach).

The third method is more in line with so-called credibility theory developed by Liu (2007) who reconstructs a PDF from a pair of possibility and necessity measures $(\Pi, N)$ as:

$$
F(x)=(\Pi(x \leq r)+N(x \leq r)) / 2 .
$$

Our third proposal for computing $F_{3}$ generalizes this procedure to belief-plausibility function pairs. 
In order to practically account for the decision-maker attitude, it is usual to introduce an optimism index $\alpha_{i}$ such that if the value of the (say) pollution index is only known to belong to $\left[a_{i}, b_{i}\right]$, the value considered as reasonably optimistic by the decision-maker is the average:

$$
f\left(a_{i}, b_{i}\right)=\alpha_{i} a_{i}+\left(1-\alpha_{i}\right) b_{i}
$$

This approach, which is based on earlier work by Hurwicz (1951), thus proposes to compute a single indicator as a weighted average of focal element bounds. It achieves a trade-off between optimistic and pessimistic estimates. In the decision theory tradition $f\left(a_{i}, b_{i}\right)$ is viewed as the certainty-equivalent of an uncertain situation whose output is $a_{i}$ with probability $\alpha_{i}$ and $b_{i}$ with probability $1-\alpha_{i}$. Under this view, the original random set is replaced by a standard probability measure $P^{2}$ obtained by assigning each probability weight $m_{i}$ to risk model output values $\alpha_{i} a_{i}+\left(1-\alpha_{i}\right) b_{i}, i=1, \ldots, n$.

In the third method, each interval $\left[a_{i}, b_{i}\right]$ would be replaced by the probability function $P^{3}{ }_{i}$ yielding $a_{i}$ with probability $\alpha_{i}$ and $b_{i}$ with probability $1-\alpha_{i}$. Its PDF can be expressed as:

$$
F_{i}(r)=\alpha_{i} \Pi_{i}(x \leq r)+\left(1-\alpha_{i}\right) N_{i}(x \leq r),
$$

where Boolean possibility and necessity measures $\Pi_{i}$ and $N_{i}$ derive from the interval $\left[a_{i}, b_{i}\right]$, following Eqns. (3) and (4). The probability measure $P^{3}$ thus obtained from the whole random set can be defined as follows: $P^{3}=\sum_{i=1, \ldots, n} m_{i} P_{i}^{3}$.

In practice, a single value $\alpha$ will be used to represent the decision-maker's attitude towards uncertainty. Then, for methods 2 and 3 we respectively get:

$$
\begin{aligned}
& F_{2}^{-1}(\lambda)=\alpha F^{*-1}(\lambda)+(1-\alpha) F_{*}^{-1}(\lambda), \forall \lambda \in(0,1] \\
& F_{3}(x)=\alpha F^{*}(x)+(1-\alpha) F_{*}(x)
\end{aligned}
$$

i.e. $F_{2}$ is obtained by taking the weighted average of upper and lower bounds of each cut of the p-box, while $F_{3}$ is obtained by the weighted average of the upper and lower fractiles. Note that the two PDFs significantly differ, but they have the same expected value:

$$
E V_{2}=E V_{3}=\alpha \sum_{i=1, \ldots, n} m_{i} a_{i}+(1-\alpha) \sum_{i=1, \ldots, n} m_{i} b_{i}
$$

However their variance is very different. In particular $F_{3}$ has a larger variance than the ones of the upper and lower distributions, while $F_{2}$ has a variance that is a trade-off between them. For instance if the probability box is an interval, the second approach suggests that randomness may be absent and $F_{2}$ proposes a substitute deterministic value. On the contrary the PDF $F_{3}$ has variance $\alpha(1-\alpha)(a-b)^{2}$. This feature makes the choice of the second (Jaffray) method preferable to the third one as $F_{2}$ has a shape more in conformity to the available information. We refer to this PDF as a "Confidence Index" in the sequel. The same expected value is also obtained when $\alpha=1 / 2$ with the pignistic transform $F_{l}$ ): 
It must be fully recognized that the choice of weight $\alpha$ is subjective. However, this subjectivity is only introduced at the decision-making step in the form of a single PDF used as a sensible reference displayed along with the pessimistic and optimistic outputs. This is very different from displaying a single distribution obtained by propagating single distributions introduced at the beginning of the risk analysis step. According to the Bayesian usage, PDFs allegedly representing the state of knowledge of experts must be selected for any parameter, even in the presence of incomplete/imprecise information. The Bayesian credo makes sense at the decision level, according to the declared intention of its founder (Savage), and the approach proposed here does not really contradict this view. It only postulates that in the end the decision is made according to expected utility of some probability function. What appears debatable is to claim that one must introduce a unique subjective probability function, each and every time incomplete information of some kind is met, while no decision is at stake (e.g. when just collecting information). Nothing in the Bayesian doctrine prescribes such an extreme view.

Our basic assumption is that the selection of this probability function by an expert makes more sense at the very end of the risk analysis chain because available information should be faithfully propagated up until that point. If this information is considered to be insufficient by the expert, he may decide to collect more. If the information is incomplete but no data collection is possible, then a scientific judgment must take place anyway, and our confidence index can contribute to it. The potential advantages of the proposed approach are illustrated in the next section.

\section{ILLUSTRATION AND DISCUSSION}

The primary objective of this application is to illustrate the use of the "Confidence Index" defined previously. The choice of the term "Confidence Index" is borrowed from the field of meteorology (WMO 2008). The meteorological community has extensive experience with respect to predicting natural events and also of communicating on these predictions with the general public. It is therefore significant that meteorologists should have adopted the term "Confidence Index" to communicate on the uncertainty relative to their predictions, as the term holds value both from a scientific and sociological viewpoint: scientific because it avoids referring to any particular uncertainty paradigm (probabilistic, possibilistic, etc.); sociological because the notion of "confidence" has positive connotations. This same term is gaining 
acceptance in other fields, for example traffic forecasting (Danech-Pajouh and Sauvadet 2003).

The illustration relies on a generic health risk calculation for the case of individual exposure to a chlorinated organic solvent (1,1,2-Trichloroethane) via the consumption of contaminated drinking water. Toxicologists consider that 1,1,2-tricholorethane is a "nothreshold”, potentially carcinogenic substance: exposure generates a risk whatever the level of exposure (e.g. EPA 1987). The chronic carcinogenic toxicological reference value for this substance is a unit excess risk (UER), namely, a probability (or expected value) of excess cancer per unit daily dose, determined based on dose-response relationships (an oral slope factor; EPA 1987). For an exposed individual, we calculate an individual excess risk (IER) as the product of the absorbed dose and the unit excess risk. The calculated excess risk can then be compared to a threshold of tolerable individual excess risk defined by the health authority.

Individual excess risk and absorbed dose are calculated from (EPA 1989):

$$
I E R=D \cdot U E R \quad \text { and: } \quad D=\frac{I \cdot C \cdot E F \cdot E D}{B W \cdot A T}
$$

where: $\mathrm{D}$ = absorbed dose (mg pollutant absorbed per Kg body weight and per day), I = quantity of water ingested per day $(\mathrm{L} / \mathrm{d}), \mathrm{C}=$ concentration of 1,1,2-trichloroethane in drinking water $(\mathrm{mg} / \mathrm{L}), \mathrm{EF}=$ exposure frequency $(\mathrm{d} / \mathrm{yr}), \mathrm{ED}=$ exposure duration $(\mathrm{yr}), \mathrm{BW}=$ body weight $(\mathrm{Kg}), \mathrm{AT}=$ averaging time (d), UER = Unit Excess Risk (expected excess cancer per unit dose; $\left.\left(\mathrm{mg} / \mathrm{Kg}^{-\mathrm{d}}\right)^{-1}\right)$, IER = Individual Excess Risk (expected excess cancer resulting from dose D).

The representation of the problem parameters requires two modes of representation described previously: probability and possibility distributions. It is assumed that there are sufficient drinking water concentration measurements $\left(C_{i}\right)$ to allow the identification of a statistically representative probability density function for this unknown quantity. Concentration in drinking water is described by a triangular probability density function of mode $10 \mu \mathrm{g} / \mathrm{l}$ and lower and upper values 5 and $20 \mu \mathrm{g} / \mathrm{l}$ respectively. It is also assumed that statistical data regarding population residence times are available such that the exposure duration (DE) can also be represented by a unique probability distribution: a triangular probability density function of mode 30 years and lower and upper limits 10 and 50 years respectively. Body weight and averaging time are taken as constants (respectively $70 \mathrm{~kg}$ and 70 years) in order to provide a generic character to the exposed individual but also to be consistent with the toxicological reference value (UER) defined for a lifelong exposure (taken as 70 years). All other parameters (rate of ingestion, exposure frequency, unit excess risk) are 
represented by triangular possibility distributions presented in Table 1, due to assumed epistemic uncertainties reflecting a lack of information. For the oral slope factor, the core is taken from EPA (1987), while the lower and the upper bounds were proposed by experts. Note that in practice, examination of the experimental data that led to the EPA recommendation may help identify more suitable values.

The individual excess risk threshold, defined by the Health Authority, is taken as $10^{-5}$ : it is an expected number of excess cancers for an exposed individual. This threshold can also be thought of as implying that the Health Authority accepts that in a population of $10^{5}$ identical individuals receiving precisely the same dose, one individual (expected value) would develop a cancer related to the exposure. We are interested in the probability of exceeding (or, conversely, remaining below) this threshold. For the hybrid calculation, fuzzy intervals were discretized into 10 cut-sets, probability distributions into 50 classes, and 100 iterations were used for the Monte Carlo random sampling. The results of the calculation are presented in Fig. 2. The distance between the $\mathrm{Pl}$ and Bel functions is a consequence of the incompleteness of information relative to the three parameters in Table 1. Also shown in Fig. 2 for comparison purposes, is the result of a Monte Carlo calculation performed assuming probability density functions for all model parameters, with total stochastic independence. Probability density functions for the three ill-informed parameters have the same shapes as the fuzzy intervals in Table 1.

With respect to the acceptability of the calculated risk, in the case of the Monte Carlo calculation the answer is quite straightforward. The probability of lying below the threshold defined by the health authority is $95 \%$, implying that there is only a $5 \%$ chance of exceeding the threshold. Such a level of risk might seem acceptable but it is reminded that the result of this calculation is biased by the fact that unique PDFs were selected in presence of incomplete information. In the case of the hybrid calculation, results suggest that the probability of lying below the threshold is comprised between 62\% (lower bound; Bel) and 100\% (upper bound; $\mathrm{Pl})$. In this case, there are two possible courses of action. One option could be to decide that the distance between the upper and lower probability bounds is too great, and therefore, the epistemic uncertainty regarding certain parameters should be reduced by performing additional measurements. But in many situations it will not be possible, for reasons of budget and time constraints, to follow this line. It is therefore proposed to compute the "Confidence Index” defined in the previous section, as shown in Fig. 3 where a weight $\alpha=1 / 3$ was used, implying that more weight $(2 / 3)$ is given to the pessimistic probability bound, than to the optimistic bound (1/3). In a context of aversion to risk, it would seem normal to privilege the 
pessimistic limit, but without completely obliterating the optimistic one. Comparison with the risk threshold of $10^{-5}$ suggests that the calculated risk is below the threshold with a Confidence Index of $80 \%$. The decision-maker must then decide whether or not this level of confidence is sufficient to accept the risk.

It may be of interest in practice to compare the results obtained by the standard Bayesian approach and the results obtained by our approach, in order to see if the subjective probability assessments on input parameters lead to an optimistic or a pessimistic view of the actual risk. It may also bring some insight for a better choice of the optimism index. However such a comparison may be delusive as the subjective input distributions are exploited as if they expressed variability (by the Monte-Carlo simulation), and the output variance will be all the smaller as many ill-informed input parameters will be handled in this way. So, while the choice of probabilistic substitute to partial knowledge may look easier to perform on input parameters (for which expertise exists) than on the risk model output itself, the reduction of uncertainty due to the probabilistic simulation technique will be more significant than if the ill-informed parameters are modelled by set-like entities propagated in the interval analysis style and a confidence index is derived from the output p-box (this is patent comparing the pure Monte-Carlo result on Figure 2 and the confidence index on Figure 3).

\section{CONCLUSION}

The way information regarding risk model parameters is represented in risk assessments should be consistent with the nature of this information. In particular the confusion between stochastic and epistemic uncertainty should be avoided so that the results of risk assessments adequately reflect available information. In this paper we first review methods for representing and propagating uncertain information in risk assessments and then propose a flowchart as an aid in the choice of tools for representing uncertain information. This flowchart highlights the idea that the important question at the data collection step is "what do I know?” rather than "what probability should I assign?” and also that no single information representation tool can be applied to all types of information.

This paper also focuses on a potential shortcoming of existing joint propagation methods in a context of decision-making, i.e. that they yield imprecise levels of probability that a (risky) proposition is true or not. Several approaches for circumventing this shortcoming are presented and one approach is selected, based on Jaffray's generalization of Hurwicz criterion to belief functions. It selects a subjective probability measure (dubbed "Confidence Index”, a name borrowed from common practice in meteorology). This probability measure, reflecting 
the decision-maker's attitude with respect to ambiguity and risk, is applied to an illustrative example. The proposed approach introduces the decision-maker subjectivity at the final decision-making stage, which is more easily justified than when modeling input information. Indeed our method does not mask epistemic uncertainty, while a full-fledged Bayesian approach to modeling all input parameters runs the risk of confusing epistemic uncertainty with stochastic variability, both being entangled in the unique distribution obtained by the propagation step. Our proposal is to represent epistemic uncertainty and stochastic variability by distinct tools, preserving this distinction after the propagation step, while offering the decision-maker a practical way to express a level of aversion to risk, thus converging to a more easily interpretable (subjective) risk probability. It is felt that the risk assessor should attempt to forward the available information to the decision-maker as faithfully as possible, so that the range of possible outcomes be known. If this range is judged too wide, then action might be taken in order to reduce uncertainties in model input parameters (e.g. via measurement). Such an analysis can never be carried out from a Monte Carlo simulation performed using postulated PDFs on input parameters, as there is no way of distinguishing, in the variance of computed output, the actual variability resulting from true stochastic randomness from apparent variability due to subjective probability judgments.

\section{ACKNOWLEDGEMENTS}

This work was supported by the $\mathrm{CO}_{2}$ Programme of the French National Research Agency (ANR; project CRISCO2, N ANR-06-CO2-003).

\section{REFERENCES}

Aven T. 2010. On the need for restricting the probabilistic analysis in risk assessments to variability. Risk Analysis, 30, 354-360.

Baccou, J., Chojnacki, E., Mercat-Rommens, C., Baudrit, C. 2008. Extending Monte Carlo simulations to represent and propagate uncertainties in presence of incomplete knowledge: Application to the transfer of a radionuclide in the environment. Journal of Environmental Engineering, 134(5), 362-368.

Baraldi, P., Zio, E. 2008. A combined Monte Carlo and possibilistic approach to uncertainty propagation in event tree analysis. Risk Analysis, 28(5), 1309-1325.

Bardossy A., Bogardi I., Kelly,W.E. 1988. Imprecise (fuzzy) information in geostatistics. Math. Geol. 20:287311 
Baudrit, C., Dubois, D. 2006. Practical representations of incomplete probabilistic knowledge. Computational Statistics \& Data Analysis, 51 86-108.

Baudrit, C., Dubois, D., Perrot N. 2008. Representing parametric probabilistic models tainted with imprecision. Fuzzy Sets and Systems, 159, 1913-1928.

Baudrit, C., Guyonnet, D., Dubois, D. 2007. Joint propagation of variability and partial ignorance in a groundwater risk assessment. Journal of Contaminant Hydrology, 93: 72-84.

Baudrit, C., Dubois, D., Guyonnet, D. 2006. Joint propagation and exploitation of probabilistic and possibilistic information in risk assessment models. IEEE Transactions on Fuzzy Systems, vol.14, No.5, pp.593-608.

Baudrit, C., Guyonnet, D., Dubois, D. 2005. Post-processing the hybrid method for addressing uncertainty in risk assessments. J. Envir. Engrg., ASCE, 131(12), 1750-1754.

Bellenfant, G., Guyonnet, D., Dubois, D., Bouc, O. 2008. Uncertainty theories applied to the analysis of CO2 plume extension during geological storage. In: GHGT-9 - 9th international conference on greenhouse gas control technologies - Washington - USA - 16-20/11/2008.

BSI 2007. Occupational health and safety management systems. Requirements. BS OHSAS 18001:2007. British Standards Institute. United Kingdom. ISBN: 9780580508028

Ben-Haim, Y. 2006 Info-Gap, Academic Press, New York.

Burton, C.S., Kim, J., Clarke, D.G., Linkov, I. 2008. A risk-informed decision framework for setting environmental windows for dredging projects. Science of the total environment, 403, 1-11.

CEC 2006. Proposal for a Directive of the European Parliament and of the Council establishing a framework for the protection of soil and amending Directive 2004/35/EC. COM 2006/0086 of the Commission of the European Communities, Brussels.

Chateauneuf A. and Cohen M. 2009. Cardinal extensions of the EU model based on Choquet integral. In D. Bouyssou, D. Dubois, M. Pirlot, and H. Prade, editors, Decision-Making Process- Concepts and Methods, chapter 3, pages 401-433. ISTE \& Wiley, London.

Chilès, J.-P., Delfiner, P. 1999. Geostatistics: Modeling Spatial Uncertainty by Jean-Paul Chilès and Pierre Delfiner, Wiley, New York, 695 pp.

Cooper, J.A., Ferson, S., Ginzburg, L. 1996. Hybrid processing of stochastic and subjective uncertainty data. Risk Anal. 16(6), 785-791.

Colyvan, M. 2008. Is probability the only coherent approach to uncertainty? Risk Analysis, 28(3), 645-652.

Connover, W. and Iman, R. 1982. A distribution-free approach to inducing rank correlation among input variables. Technometric, 3, 311-334.

Couso, I., Moral, S., Walley, P. 2000. A survey of concepts of independence for imprecise probabilities. Risk Decision and Policy, 5, 165-181. 
Danesh-Pajouh, M., Sauvadet, V. 2003. A statistical consistency method for evaluating the output from traffic simulation and forecasting methods. In: IEEE International Conference on Systems, Man and Cybernetics, 4(5-8), 4021-4026.

Dempster, A.P. 1967. Upper and lower probabilities induced by a multivalued mapping. Annals of Mathematical Statistics, 38, 325-339.

Destercke S., Dubois D., Chojnacki E. 2008. Unifying practical uncertainty representations - Part I: Generalized p-boxes. International Journal of Approximate Reasoning, 49(3), 649-663.

Dubois, D. 2006. Possibility theory and statistical reasoning. Computat. Statistics \& Data Analysis, 51, 47-69.

D. Dubois 2010, Representation, Propagation, and Decision Issues in Risk Analysis Under Incomplete Probabilistic Information, Risk Analysis, 30 p 361-368.

Dubois, D., Prade, H. Smets P. 1996. Representing partial ignorance. IEEE Trans. on Systems, Man and Cybernetics, 26(3), 361-377.

Dubois, D., Prade, H. 1992. When upper probabilities are possibility measures. Fuzzy Sets and Systems, 49, 6574.

Dubois, D., Prade, H. 1988. Possibility Theory: An Approach to Computerized Processing of Uncertainty, Plenum Press, New York.

Dubois, D., Prade, H. 2009. Formal representations of uncertainty. Decision-making Process- Concepts and Methods. Denis Bouyssou, Didier Dubois, Marc Pirlot, Henri Prade (Eds.), ISTE London \& Wiley, Chap. 3, p. $85-156$.

Dubois, D., Kerre E., Mesiar R., Prade H. 2000. Fuzzy interval analysis. In: Fundamentals of Fuzzy Sets, Dubois,D. Prade,H., Eds: Kluwer, Boston, Mass, The Handbooks of Fuzzy Sets Series, 483-581.

Ellsberg, D. 1961. Risk, ambiguity, and the Savage axioms. The Quarterly Journal of Economics, 643-669.

EPA 1989. Risk Assessment Guidance for Superfund. Volume I. Human Health Evaluation Manual (Part A). EPA/540/1-89/002, US EPA, Washington, USA.

EPA 1987. Drinking Water Health Advisory for 1,1,2-Trichloroethane. Prepared by the Office of Health and Environmental Assessment, Environmental Criteria and Assessment Office, Cincinnati, OH for the Office of Drinking Water, Washington, DC. ECAO-CIN-W027.

Ferson, S., Ginzburg, L., Kreinovich, V., Myers, D.M., Sentz, K. 2003. Construction of probability boxes and Dempster-Shafer structures. Sandia National Laboratories Technical report SANDD2002-4015.

Ferson, S., Ginzburg, L.R. 1996. Different methods are needed to propagate ignorance and variability. Reliability Engineering and Systems Safety, 54, 133-144.

Ferson, S. 1996. What Monte Carlo methods cannot do. Human and Environmental Risk Assessment, 2, 9901007.

Gilboa I. and Schmeidler D. 1989. Maxmin expected utility with a non-unique prior. Journal of Mathematical Economics, 18:141-153. 
Guyonnet, D., Bourgine, B., Dubois, D., Fargier, H., Côme, B., Chilès, J.P. 2003. Hybrid approach for addressing uncertainty in risk assessments. J. Envir. Engrg., ASCE, 129, 68-78.

Guyonnet, D., Côme, B., Perrochet, P., Parriaux, A. 1999 - Comparing two methods for addressing uncertainty in risk assessments. Journal of Environmental Engineering, 125 :7, 660-666.

Hanss M., , 2004. Applied Fuzzy Arithmetic. Berlin, Germany: Springer.

Helton, J.C., Johnson, J.D., Oberkampf, W.L. 2004. An exploration of alternative approaches to the representation of uncertainty in model predictions. Reliability Engineering System Safety, 85(1-3), 39-71.

Hoffman, F.O., Hammonds, J.S. 1994. Propagation of uncertainty in risk assessments: the need to distinguish between uncertainty due to lack of knowledge and uncertainty due to variability. Risk Analysis, 14, 707-712.

Herstein, I.N., Milnor, J. 1953. An axiomatic approach to measurable utility. Econometrica, 21, 291-297.

Huber, P. J. 1981. Robust Statistics, Wiley, New York.

Hurwicz, L. 1951. Optimality criteria for decision making under ignorance. Cowles Commission discussion paper, Statistics No. 370 .

Jaffray, J.-Y. 1988. Linear utility theory for belief functions. Operations Research Letters, 1988.

Jaffray, J.-Y. 1994. Dynamic decision making with belief functions. In: advances in the Dempster-Shafer theory of evidence. Yager, R., Kacprzyk, J., Fedrizzi, M (Editors). John Wiley \& Sons, New York.

Kaufmann, A., Gupta, M.M. 1985. Introduction to Fuzzy Arithmetic: Theory and Applications. Van Nostrand Reinhold Company, New York, USA.

Kendall, D.G. 1974. Foundations of a theory of random sets. In Stochastic Geometry (E.F. Harding and D.G. Kendall, editors): 322-376. J.Wiley, New York.

Kentel, E. 2006. Uncertainty modeling in health risk assessment and groundwater resources management. Thesis of the Georgia Institute of Technology, USA, 339 pp.

Kentel, E., Aral, M.M. 2005. 2D Monte Carlo versus 2D fuzzy Monte Carlo health risk assessment. Stoch. Environ. Res. Risk Assess., 19, 86-96.

Kriegler, E., Held, H. 2005. Utilizing belief functions for the estimation of future climate change International Journal of Approximate Reasoning, 39(2-3), 185-209.

Li., H.L., Huang, G.H., Zou, Y. 2008. An integrated fuzzy-stochastic modelling approach for assessing healthimpact risk from air pollution. Stoch. Environ. Res. Risk Assess. 22, 789-803.

Li, J., Huang, G.H., Zeng, G.M., Maqsood, I., Huang, Y.F. 2007. An integrated fuzzy-stochastic modeling approach for risk assessment of groundwater contamination, Journal of Environmental Management (Elsevier), 82(2), 173-188.

Lindley, D.V. 1971. Making Decisions. Wiley-Interscience.

Liu, B.D. 2007. Uncertainty Theory, 2nd. Ed., Springer. 
Loquin, K. Dubois D. 2010. Kriging and epistemic uncertainty : a critical discussion. To appear in Methods for Handling Imperfect Spatial Information. Eds: R. Jeansoulin, O. Papini, H. Prade and S. Schockaert. Springer Verlag.

von Neumann J., Morgenstern. O. 1947. Theory of games and economic behavior. Princeton University Press.

OJEC (2000). Directive 2000/60/EC of the European Parliament and of the Council of 23 October 2000 establishing a framework for community action in the field of water policy. Official Journal of the European Communities, 22 december 2000, L327.

OJEC 2008. Directive 2008/98/EC of the European Parliament and of the Council of 19 November 2008 on waste and repealing certain Directives. Official Journal of the European Communities, 22 November, L $312 / 3$.

Pollard, S.J., Yearsley, R., Reynard, N., Meadowcroft, I.C., Duarte-Davidson, R., Duerden, S. 2001. Current directions in the practice of environmental risk assessment in the United Kingdom. Environ. Sci. Tech. 36(4), 530-538.

Regan, H.M., Ferson, S., Berleant, D. 2004. Equivalence of methods for uncertainty propagation of real-valued random variables. Int. J. Approx. Reasoning 36(1), 1-30.

Savage, L. 1954. The foundations of statistics. New York. Wiley, (2nd Edition 1972, Dover).

Shafer, G. 1976. A Mathematical Theory of Evidence. Princeton University Press.

Smets, P. Decision making in the TBM: the necessity of the pignistic transformation. Int. J. Approx. Reasoning 38(2): 133-147 (2005)

Troffaes M. 2007. Decision making under uncertainty using imprecise probabilities. Int. J. Approx. Reasoning, 45(1):17-29.

Vegter, J. 2001. Sustainable contaminated land management: a risk-based land management approach. Land Contamination \& Reclamation, 9(1), 95-100.

Walley, P. 1991. Statistical reasoning with imprecise probabilities. Chapman and Hall.

Williamson, R.C., Downs, T. 1990. Probabilistic arithmetic: Numerical methods for calculating convolutions and dependency bounds. Int. Jour. of Approx. Reason., 4(2), 89-158.

WMO 2008. Guidelines on communicating forecast uncertainty. World Meteorological Organization, WMO/TD 4122.

Zadeh, L. 1978. Fuzzy sets as a basis for a theory of possibility. Fuzzy Sets and Systems, 1, pp.3-28. 


\section{List of Tables and Figures}

Table 1. Parameter values used for the illustration

\begin{tabular}{llllll}
\hline Parameter & Unit & Mode of & $\begin{array}{l}\text { Lower } \\
\text { representation }\end{array}$ & $\begin{array}{l}\text { Mode or } \\
\text { limit }\end{array}$ & Upper limit \\
\hline Concentration in water & $\mu \mathrm{g} / \mathrm{L}$ & Probability & 5 & 10 & 20 \\
Ingestion & $\mathrm{L} / \mathrm{d}$ & Fuzzy interval & 1 & 1.5 & 2.5 \\
Exposure frequency & $\mathrm{d} / \mathrm{year}$ & Fuzzy interval & 200 & 250 & 350 \\
Exposure duration & Years & Probability & 10 & 30 & 50 \\
Oral slope factor & $(\mathrm{mg} / \mathrm{Kg} / \mathrm{d})^{-1}$ & Fuzzy interval & $2 \times 10^{-2}$ & $5.7 \times 10^{-2}$ & $10^{-1}$ \\
\hline
\end{tabular}


Fig. 1. Flowchart relating information nature to mode of information representation

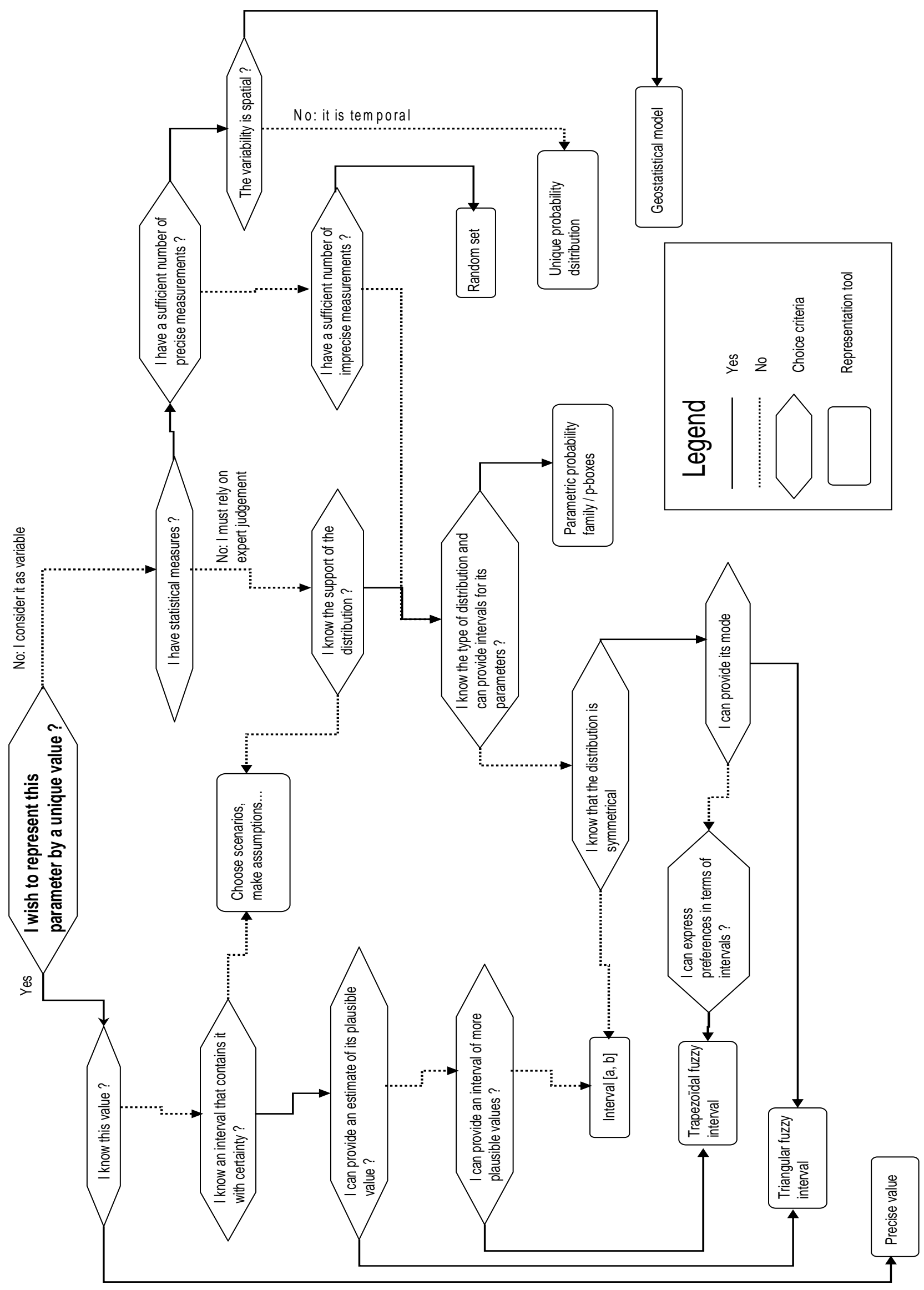


Fig. 2. Result of the application risk calculation

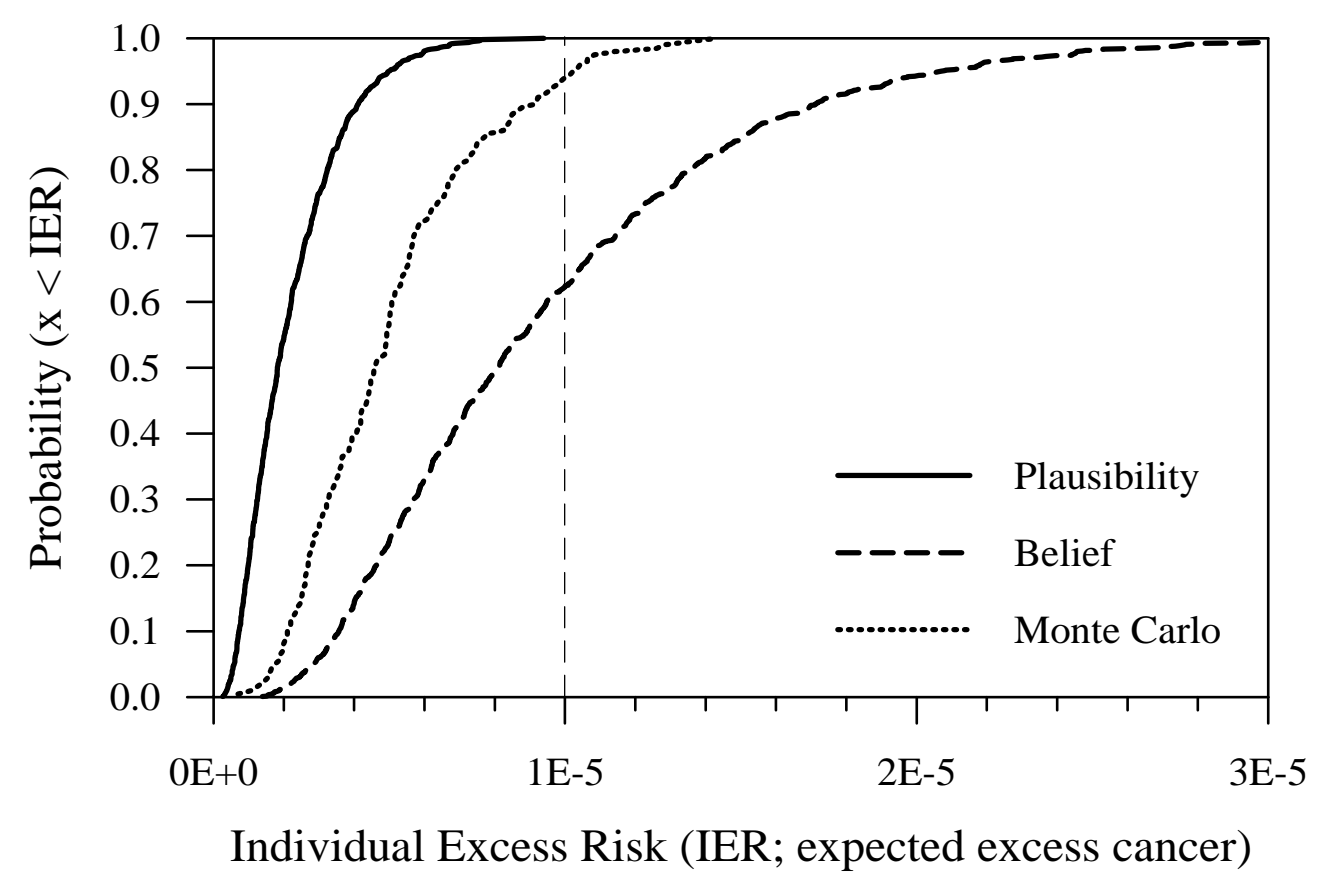

Fig. 3. Illustration of the confidence index for a value $\alpha=1 / 3$

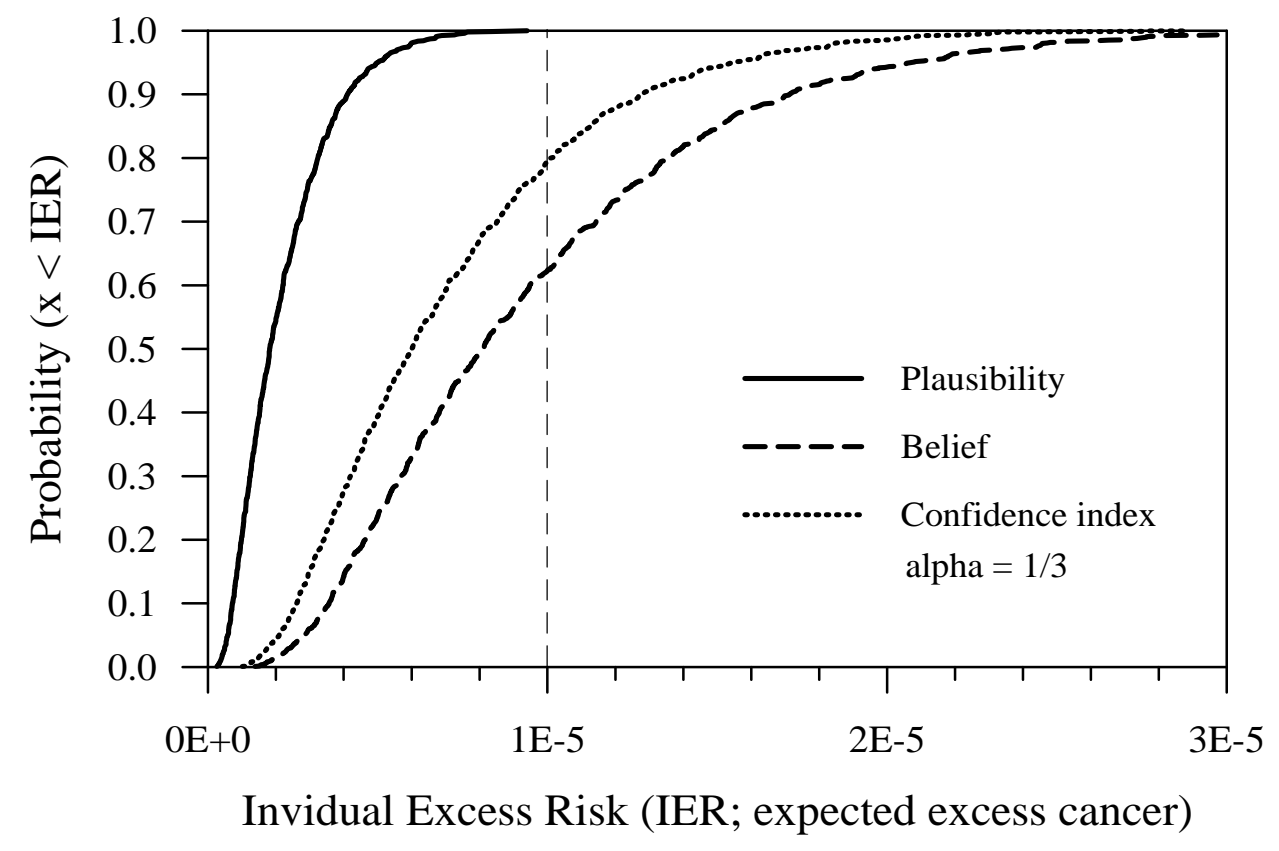

